\title{
OPERATOR-VALUED FEYNMAN INTEGRALS OF CERTAIN FINITE-DIMENSIONAL FUNCTIONALS
}

\author{
G. W. JOHNSON AND D. L. SKOUG
}

\begin{abstract}
Let $C_{0}[a, b]$ denote the space of continuous functions $x$ on $[a, b]$ such that $x(a)=0$. Let $F(x)=f_{1}\left(x\left(t_{1}\right)\right) \cdots f_{n}\left(x\left(t_{n}\right)\right)$ where $a=t_{0}<t_{1}<\cdots<t_{n}=b$. Recently, Cameron and Storvick defined an operator-valued "Feynman Integral." In their setting, we give a strong existence theorem as well as an explicit formula for the "Feynman Integral" of functionals $F$ as above under weak restrictions on the $f_{j}$ 's. We also give necessary and sufficient conditions for the operator to be invertible and an explicit formula for the inverse.
\end{abstract}

1. Introduction and notation. Let $\mathscr{L}\left(L_{2}\right)$ be the space of bounded linear operators on $L_{2}=L_{2}(-\infty, \infty)$. Let $B[a, b]$ be the space of functions on $[a, b]$ which are continuous except for a finite number of finite jump discontinuities.

We need the following definitions of Cameron and Storvick [2]. (The definitions are not intended to imply the existence of the operators involved. In fact the main theorems of [2] give various conditions on $F$ insuring the existence of these operators.) Let $\psi \in L_{2}$, $\xi \in(-\infty, \infty)$, and $F$ a functional on $B[a, b]$. For $\lambda>0, I_{\lambda}(F)$ is the operator on $L_{2}$ defined by the Wiener in tegral

$$
\left(I_{\lambda}(F) \psi\right)(\xi)=\int_{C_{0}[a, b]} F\left(\lambda^{-1 / 2} x+\xi\right) \psi\left(\lambda^{-1 / 2} x(b)+\xi\right) d x .
$$

For $\operatorname{Re} \lambda>0, I_{\lambda}^{\text {an }}(F)$ is defined to be the operator-valued function of $\lambda$ which agrees with $I_{\lambda}(F)$ for $\lambda>0$ and is analytic throughout $\operatorname{Re} \lambda>0$. For $\operatorname{Re} \lambda>0$ and any partition $\sigma: a=s_{0}<s_{1}<\cdots<s_{n}=b$ the operator $I_{\lambda}^{\sigma}(F)$ is defined by the formula

$$
\begin{aligned}
& \left(I_{\lambda}^{\sigma}(F) \psi\right)(\xi)=\lambda^{n / 2}\left[(2 \pi)^{n}\left(s_{1}-a\right) \cdots\left(s_{n}-s_{n-1}\right)\right]^{-1 / 2} \int_{-\infty}^{\infty} \cdot(n) \\
& \cdot \int_{-\infty}^{\infty} \psi\left(v_{n}\right) \cdot f_{\sigma}\left(\xi, v_{1}, \cdots, v_{n}\right) \exp \left(-\sum_{j=1}^{n} \frac{\lambda\left(v_{j}-v_{j-1}\right)}{2\left(s_{j}-s_{j-1}\right)}\right) d v_{1} \cdots d v_{n}
\end{aligned}
$$

Received by the editors June 24, 1969.

AMS Subject Classifications. Primary 2825, 4690.

Key Words and Phrases. Feynman integral, Wiener integral, convolution operator, Fourier transform, operator valued Feynman integral. 
where $v_{0}=\xi, f_{\sigma}\left(v_{0}, v_{1}, \cdots, v_{n}\right)=F\left[z\left(\sigma, v_{0}, v_{1}, \cdots, v_{n}, \cdot\right)\right]$ and

$$
\begin{aligned}
z\left(\sigma, v_{0}, v_{1}, \cdots, v_{n}, s\right) & =v_{j} & & \text { if } s_{j} \leqq s<s_{j+1}, j=0,1, \cdots, n-1 \\
& =v_{n} & & \text { if } s=b .
\end{aligned}
$$

(If $n$ is odd we always choose $\lambda^{1 / 2}$ with nonnegative real part.) For $\operatorname{Re} \lambda>0$, the operator $I_{\lambda}^{\text {sea }}(F)$ is defined by

$$
I_{\lambda}^{\text {seq }}(F)=w \lim _{\text {norm } \sigma \rightarrow 0} I_{\lambda}^{\sigma}(F)
$$

where $w$ lim means the limit with respect to the weak operator topology on $\mathscr{L}\left(L_{2}\right)$. In case both $I_{\lambda}^{\text {an }}(F)$ and $I_{\lambda}^{\text {seq }}(F)$ exist and agree we will denote their common value by $I_{\lambda}(F)$. Finally for $\lambda=-i q, q \in(-\infty, \infty)$, $q \neq 0$, the operators $J_{q}^{\text {an }}(F)$ and $J_{q}^{\text {seq }}(F)$ are defined by

$$
J_{q}^{\mathrm{an}}(F)=w \lim _{p \rightarrow 0^{+}} I_{p-i q}^{\mathrm{an}}(F) \text { and } J_{q}^{\mathrm{seq}}(F)=w \lim _{p \rightarrow 0^{+}} I_{p-i q}^{\mathrm{seq}}(F) .
$$

Again if both exist and agree we denote their common value by $J_{q}(F)$.

Throughout the rest of this paper we assume that $F$ has the special form given in the abstract, where each $f_{j}$ is measurable and satisfies $\left\|f_{j}\right\|_{\infty}<\infty$. This restriction on the $f_{j}$ 's is much weaker than in $[1$, pp. 333-348] and [5, pp. 177-185] where Cameron's earlier definition of the Feynman and related integrals was employed to study functionals of the same type.

The main theorem below does more than establish the existence of $J_{q}(F)$ and give a formula for it. First, it shows that $J_{q}(F)$ is the strong operator limit of $I_{\lambda}(F)$ rather than just the weak operator limit; secondly, the approach of $\lambda$ to $-i q$ is restricted only to the open right half plane and not to the line $p-i q$. We mention also that the existence of $J_{q}(F)$ is established for every $q \neq 0$. In [2], where more complicated functionals $F$ are dealt with, the theorems give existence of $J_{q}(F)$ for almost every $q$, but for specific $q$, one cannot tell whether $J_{q}(F)$ exists or not.

We will see below that $I_{\lambda}(F)$ and $J_{q}(F)$ are compositions of multiplication and convolution operators. In the lemma we study the appropriate convolution operators. We also use the following notation:

$$
\int_{-\infty}^{\infty} f(u, y) d u \equiv \underset{A \rightarrow \infty}{\lim . \mathrm{m} .} \int_{-A}^{A} f(u, v) d u
$$

which means 


$$
\left.\lim _{A \rightarrow \infty} \int_{-\infty}^{\infty}\right|^{(y)} \int_{-\infty}^{\infty} f(u, y) d u-\left.\int_{-A}^{A} f(u, y) d u\right|^{2} d y=0 .
$$

2. Lemma. (a) Let

$$
\left(U_{q} \psi\right)(y)=(-i q / 2 \pi)^{1 / 2} \int_{-\infty}^{(y)} \exp \left(\frac{q i(y-x)^{2}}{2}\right) \psi(x) d x
$$

for $\psi \in L_{2}, y \in(-\infty, \infty)$ and real $q \neq 0 . U_{q}$ is a unitary operator on $L_{2}$ and $U_{q}^{\#}=U_{q}^{-1}=U_{-q}$ where $U_{q}^{\sharp}$ denotes the adjoint of $U_{q}$.

(b) Let

$$
\left(C_{\lambda} \psi\right)(y)=(\lambda / 2 \pi)^{1 / 2} \int_{-\infty}^{\infty} \exp \left(\frac{-\lambda(y-x)^{2}}{2}\right) \psi(x) d x
$$

for $\psi \in L_{2}, y \in(-\infty, \infty)$ and $\operatorname{Re} \lambda>0 . C_{\lambda}$ is in $\&\left(L_{2}\right)$, it is one-to-one, its range is contained in the set of equivalence classes of $L_{2}$ which contain a continuous function, and $\left\|C_{\lambda}\right\|=1$.

Proof. (a) The fact that $U_{q}$ is an isometry in $\mathscr{L}\left(L_{2}\right)$ is shown in Lemma 1 of [2], hence to show $U_{q}$ unitary, we only need to show that it is onto. Now

$$
\left(U_{q} \psi\right)(y)=(-i q / 2 \pi)^{1 / 2} \exp \left(i q y^{2} / 2\right) F\left[\exp \left(i q x^{2} / 2\right) \psi(x)\right](q y)
$$

where $\mathcal{F}$ denotes the $L_{2}$-Fourier transform. Thus to show $U_{q}$ onto it suffices to show that, given an $L_{2}$ function $\phi(y)$, there exists an $L_{2}$ function $\psi$ such that

$$
\mathcal{F}\left[\exp \left(i q x^{2} / 2\right) \psi(x)\right](q y)=(2 \pi /-i q)^{1 / 2} \exp \left(-i q y^{2} / 2\right) \phi(y) .
$$

This follows since the maps $\psi(x) \rightarrow \exp \left(i q x^{2} / 2\right) \psi(x)$ and $\mathcal{F}$ are both onto $L_{2}$ and the $q$ appearing in the argument just brings about a change of scale.

Since $U^{-1}=U^{\sharp}$ holds for any unitary operator, we need simply show $U_{q}^{\sharp}=U_{-q}$. Now the space $C_{K}$ of continuous functions on $(-\infty, \infty)$ with compact support is dense in $L_{2}$ and so to show $U_{q}^{\#}$ $=U_{-q}$ it suffices to show $\left(U_{q} \psi, \phi\right)=\left(\psi, U_{-q} \phi\right)$ for $\psi, \phi \in C_{K}$. This follows from the Fubini Theorem since the integrand in each case is dominated by the integrable function $|q|^{1 / 2}|\psi(x)||\phi(y)|$.

(b) $C_{\lambda}$ is one-to-one for $\left(C_{\lambda} \psi\right)(y)=0$ a.e. implies $\psi(x)=0$ a.e. since the Fourier transform of a convolution is the product of the Fourier transforms and the Fourier transform of $\exp \left(-\lambda x^{2} / 2\right)$ never vanishes. For any $\psi \in L_{2},\left(C_{\lambda} \psi\right)(y)$ is continuous since the convolution of $L_{2}$ functions is continuous.

In Lemma 1 of [2], Cameron and Storvick show that $\left\|C_{\lambda}\right\| \leqq 1$. Using a comment in [3, p. 1045] one can show quite easily that $\left\|C_{\lambda}\right\|=1$. However, the following elementary proof seems more in- 
structive. Let $\frac{1}{2}<r<k<1$ be given. It suffices to find $\psi \in L_{2}$ such that $\|\psi\|=1$ and $\left\|C_{\lambda} \psi\right\|>r$. Let $\sigma^{2}=2|\lambda|{ }^{-2} \operatorname{Re} \lambda$. Now choose integers $m$ and $n$ such that $\left(2 \pi \sigma^{2}\right)^{-1 / 2} \int_{-m \sigma}^{m \sigma} \exp \left(-x^{2} / 2 \sigma^{2}\right) d x>k$ and $(n-m \sigma) k / n$ $>r$. Let $\psi(x)=(2 n)^{-1 / 2} \chi_{(-n, n)}(x)$. Then $\|\psi\|=1$, and by use of the formula

$$
\begin{aligned}
\int_{-\infty}^{\infty} \exp \left(\frac{-\lambda(x-\xi)^{2}}{2} \frac{-\bar{\lambda}(u-\xi)^{2}}{2}\right) d \xi & \\
& =(\pi / \operatorname{Re} \lambda)^{1 / 2} \exp \left(\frac{-|\lambda|^{2}(x-y)^{2}}{4 \operatorname{Re} \lambda}\right) .
\end{aligned}
$$

We obtain

$$
\begin{aligned}
\left\|C_{\lambda} \psi\right\|^{2} & =\left(2 \pi \sigma^{2}\right)^{-1 / 2} \int_{-\infty}^{\infty} \int_{-\infty}^{\infty} \psi(u) \psi(x) \exp \left(\frac{-(x-u)^{2}}{2 \sigma^{2}}\right) d x d u \\
& \geqq\left(2 \pi \sigma^{2}\right)^{-1 / 2}(2 n)^{-1} \int_{-n}^{n} \int_{u-n}^{u+n} \exp \left(-s^{2} / 2 \sigma^{2}\right) d s d u \\
& \geqq(2 n)^{-1} \int_{-n+m \sigma}^{n-m \sigma} \int_{u-n}^{u+n}\left(2 \pi \sigma^{2}\right)^{-1 / 2} \exp \left(-s^{2} / 2 \sigma^{2}\right) d s d u \\
& \geqq(2 n)^{-1} \int_{-n+m \sigma}^{n-m \sigma} \int_{-m \sigma}^{m \sigma}\left(2 \pi \sigma^{2}\right)^{-1 / 2} \exp \left(-s^{2} / 2 \sigma^{2}\right) d s d u \\
& \geqq(2 n-2 m \sigma) k / 2 n>r>r^{2} .
\end{aligned}
$$

The following proposition establishes the existence of the operator $I_{\lambda}(F)$ for the class of $F$ 's we are considering.

Proposition. $I_{\lambda}(F)$ exists for all $\lambda$ such that $\operatorname{Re} \lambda>0$ and is given by

$$
\begin{aligned}
\left(I_{\lambda}(F) \psi\right)(\xi) & =\lambda^{n / 2}\left[(2 \pi)^{n}\left(t_{1}-a\right) \cdots\left(t_{n}-t_{n-1}\right)\right]^{-1 / 2} \\
& \cdot \int_{-\infty}^{\infty}(n) \cdot \int_{-\infty}^{\infty} \psi\left(v_{n}\right) \cdot f_{1}\left(v_{1}\right) \cdots f_{n}\left(v_{n}\right) \\
& \cdot \exp \left(-\sum_{j=1}^{n} \frac{\lambda\left(v_{j}-v_{j-1}\right)^{2}}{2\left(t_{j}-t_{j-1}\right)}\right) d v_{1} \cdots d v_{n}
\end{aligned}
$$

where $v_{0} \equiv \xi$ and $t_{0} \equiv a$.

Proof. Let $K_{\lambda}(F)$ denote the operator defined by the right-hand side of (3). In order to show that $I_{\lambda}^{\text {sea }}(F)$ exists and equals $K_{\lambda}(F)$ it suffices to show that 


$$
\lim _{\text {norm } \sigma \rightarrow 0}\left(I_{\lambda}^{\sigma}(F) \psi, \phi\right)=\left(K_{\lambda}(F) \psi, \phi\right)
$$

for all $\psi, \phi \in L_{2}$ where $I_{\lambda}^{\sigma}(F)$ is given by equation (2). This follows since for any partition $\sigma: a=s_{0}<s_{1}<\cdots<s_{m}=b$ with norm $\sigma$ $<\min \left\{t_{1}-a, \cdots, t_{n}-t_{n-1}\right\}$ we obtain

$$
\begin{aligned}
\left(I_{\lambda}^{\sigma}(F) \psi\right)(\xi) & =\lambda^{n / 2}\left[(2 \pi)^{n}\left(r_{1}-a\right) \cdots\left(r_{n}-r_{n-1}\right)\right]^{-1 / 2} \\
& \cdot \int_{-\infty}^{\infty}(n) \cdot \int_{-\infty}^{\infty} \psi\left(v_{n}\right) \cdot f_{1}\left(v_{1}\right) \cdots f_{n}\left(v_{n}\right) \\
& \cdot \exp \left(-\sum_{j=1}^{n} \frac{\lambda\left(v_{j}-v_{j-1}\right)^{2}}{2\left(r_{j}-r_{j-1}\right)}\right) d v_{1} \cdots d v_{n}
\end{aligned}
$$

by carrying out $m-n$ integrations on the right side of equation (2), where $v_{0} \equiv \xi, r_{0} \equiv a$ and $r_{j}$ is that $s_{k}$ such that $s_{k} \leqq t_{j}<s_{k+1}$. Then as norm $\sigma \rightarrow 0, r_{j} \rightarrow t_{j}^{-}$and so the result follows from the dominated convergence theorem.

For $\lambda>0, K_{\lambda}(F)$ agrees with the Wiener integral given in equation (1) and is analytic for $\operatorname{Re} \lambda>0\left[2\right.$, p. 533], and so $I_{\lambda}^{\text {an }}(F)$ exists and equals $K_{\lambda}(F)$.

THEOREM. $J_{q}(F)$ exists for all real $q \neq 0$ and is given by the right-hand side of (3) where $\lambda=-i q$ and the integrals are interpreted in the mean. In fact $J_{q}(F)$ is the strong operator limit of $I_{\lambda}(F)$ as $\lambda \rightarrow-i q$ in the righthalf plane.

Proof. We will establish that the operator defined by the righthand side of (3) (with $\lambda=-i q$ ), which we will temporarily denote by $K_{q}(F)$, is the strong operator limit of $I_{\lambda}(F)$ as $\lambda \rightarrow-i q$ in the right half plane; the first statement then follows. Careful examination of $I_{\lambda}(F)$ reveals that it is the composition of multiplication operators and convolution operators $[2$, p. 535$]$; i.e. $I_{\lambda}(F)=C_{1, \lambda} \circ M_{1} \circ \cdots$ $\circ C_{n, \lambda} \circ M_{n}$ where $C_{j, \lambda}$ and $M_{j}$ are the elements of $\mathfrak{L}\left(L_{2}\right)$ defined respectively by

$$
\left(C_{j, \lambda} \psi\right)(y)=\left(\frac{\lambda}{2 \pi\left(t_{j}-t_{j-1}\right)}\right)^{1 / 2} \int_{-\infty}^{\infty} \exp \left(\frac{-\lambda(y-x)^{2}}{2\left(t_{j}-t_{j-1}\right)}\right) \psi(x) d x
$$

and $\left(M_{j} \psi\right)(y)=\psi(y) f_{j}(y)$. Similarly $K_{q}(F)=U_{1} \circ M_{1} \circ \cdots \circ U_{n} \circ M_{n}$ where

$$
\left(U_{j} \psi\right)(y)=\left(\frac{-i q}{2 \pi\left(t_{j}-t_{j-1}\right)}\right)^{1 / 2} \quad \int_{-\infty}^{(y)} \exp \left(\frac{i q(y-x)^{2}}{2\left(t_{j}-t_{j-1}\right)}\right) \psi(x) d x .
$$


Now the map $(A, B) \rightarrow A \circ B$ of $L_{2} \times L_{2}$ to $L_{2}$ is continuous in the strong operator topology as long as $A$ is restricted to lie in a bounded subset of $\mathcal{L}\left(L_{2}\right)$ [3, p. 512]. Since $\left\|C_{j, \lambda}\right\|=1$ it will suffice to show that $C_{j, \lambda} \rightarrow U_{j}$ (s.o.) where the s.o. refers to the fact that the convergence is with respect to the strong operator topology. This suffices since if $C_{n, \lambda} \rightarrow U_{n}$ (s.o.) then $C_{n, \lambda} \circ M_{n} \rightarrow U_{n} \circ M_{n}$ (s.o.) and $M_{n-1} \circ C_{n, \lambda} \circ M_{n}$ $\rightarrow M_{n-1} \circ U_{n} \circ M_{n}$ (s.o.) and $C_{n-1, \lambda} \circ M_{n-1} \circ C_{n, \lambda} \circ M_{n} \rightarrow U_{n-1} \circ M_{n-1}$ ○ $U_{n} \circ M_{n}$ (s.o.), etc. In showing $C_{j, \lambda} \rightarrow U_{j}$ (s.o.) it clearly suffices to consider the case where $t_{j}-t_{j-1}=1$. Hence, in our notation, $U_{j}=U_{q}$ and $C_{j, \lambda}=C_{\lambda}$. Now to show $C_{\lambda} \rightarrow U_{q}$ (s.o.), it suffices to show $\left\|C_{\lambda} \psi-U_{a} \psi\right\| \rightarrow 0$ for $\psi \in C_{K}$ since for $\psi_{0} \in L_{2}$ we have the inequalities

$$
\begin{aligned}
\left\|C_{\lambda} \psi_{0}-U_{q} \psi_{0}\right\| & \leqq\left\|\left(C_{\lambda}-U_{q}\right)\left(\psi_{0}-\psi\right)\right\|+\left\|C_{\lambda} \psi-U_{q} \psi\right\| \\
& \leqq 2\left\|\psi_{0}-\psi\right\|+\left\|C_{\lambda} \psi-U_{q} \psi\right\| .
\end{aligned}
$$

Now to show $\left\|C_{\lambda} \psi-U_{q} \psi\right\| \rightarrow 0$, it suffices [4, p. 11] to show (a) $C_{\lambda} \psi \rightarrow U_{q} \psi$ weakly and (b) $\left\|C_{\lambda} \psi\right\| \rightarrow\left\|U_{q} \psi\right\|$. To obtain (a), it suffices to show $\left(C_{\lambda} \psi, \phi\right) \rightarrow\left(U_{q} \psi, \phi\right)$ for $\phi \in C_{K}$; one may see this by an inequality similar to (4). Since $\left(C_{\lambda} \psi, \phi\right)$ is a complex-valued function, it suffices to show $\left(C_{\lambda_{m}} \psi, \phi\right) \rightarrow\left(U_{q} \psi, \phi\right)$ along any sequence $\lambda_{m} \rightarrow-i q$ with $\operatorname{Re} \lambda_{m}>0$. But as $\psi, \phi \in C_{K},|\psi \phi|$ is integrable over $(-\infty, \infty) \times(-\infty, \infty)$ and so the result follows upon application of the Fubini Theorem and the dominated convergence theorem.

To establish (b) it again suffices to show $\left\|C_{\lambda_{m}} \psi\right\| \rightarrow\left\|U_{q} \psi\right\|$ for any sequence $\lambda_{m} \rightarrow-i q$ with $\operatorname{Re} \lambda_{m}>0$. Now since $\left\|C_{\lambda_{m}}\right\|=1$ and $U_{q}$ is an isometry, we have lim inf $\left\|C_{\lambda_{m}} \psi\right\| \leqq \lim \sup \left\|C_{\lambda_{m}} \psi\right\| \leqq\|\psi\|=\left\|U_{q} \psi\right\|$. But also, since balls about zero in $L_{2}$ are compact in the weak topology, only finitely many of the $C_{\lambda_{m}}$ can be in any such ball of radius less than $\left\|U_{q} \psi\right\|$. Hence $\left\|U_{q} \psi\right\| \leqq \lim \inf \left\|C_{\lambda_{m}} \psi\right\|$ and so $\lim \left\|C_{\lambda_{m}} \psi\right\|=\left\|U_{q} \psi\right\|$ which completes the proof of the theorem.

Corollary. $J_{q}(F)$ is invertible as an element in $\mathscr{L}\left(L_{2}\right)$ if and only if each $f_{j}$ is bounded away from zero a.e. In this case $J_{q}(F)^{-1}$ is given by the formula

$$
\begin{aligned}
\left(J_{q}(F)^{-1} \psi\right)(\xi)= & (i q)^{n / 2}\left[(2 \pi)^{n}\left(t_{1}-a\right) \cdots\left(t_{n}-t_{n-1}\right)\right]^{-1 / 2} \\
& \cdot \int_{-\infty}^{\infty}(n) \cdot{ }^{\left(v_{n-1}\right)} \int_{-\infty}^{\infty} \psi\left(v_{n}\right) \\
& \cdot\left[f_{n}(\xi) f_{n-1}\left(v_{1}\right) f_{n-2}\left(v_{2}\right) \cdots f_{1}\left(v_{n-1}\right)\right]^{-1} \\
& \cdot \exp \left(\frac{-i q}{2} \sum_{j=1}^{n} \frac{\left(v_{n-j+1}-v_{n-j}\right)^{2}}{\left(t_{j}-t_{j-1}\right)}\right) d v_{n} \cdots d v_{1}
\end{aligned}
$$

where $v_{0} \equiv \xi$ and $t_{0} \equiv a$. 
Proof. It is easily verified $[4$, p. 32$]$ that $M_{j}$ is invertible if and only if $f_{j}$ is bounded away from zero a.e., and, in this case, $M_{j}^{-1}$ is multiplication by $1 / f_{j}$. Thus under the conditions of the corollary, it follows from the lemma and theorem above that $J_{q}(F)^{-1}=M_{n}^{-1} \circ U_{n}^{-1}$ $\circ \cdots \circ M_{1}^{-1} \circ U_{1}^{-1}$ exists and is given by (5). If $J_{q}(F)$ is not invertible, some $M_{j}$ must fail to be invertible by the lemma and so $f_{j}$ is not bounded away from zero a.e.

ACKNowledgement. The second author wishes to thank the University of Nebraska Research Council for financial support.

\section{BIBLIOGRAPHY}

1. R. H. Cameron, The Ilstow and Feynman integrals, J. Analyse Math. 10 (1962 /63), 287-361. MR 27 \#831.

2. R. H. Cameron and D. A. Storvick, An operator valued function space integral and a related integral equation, J. Math. Mech. 18 (1968), 517-552.

3. N. Dunford and J. Schwartz, a) Linear operators. I: General theory, Pure and Appl. Math., vol. 7, Interscience, New York, 1958. MR 22 \#8302. b) Linear operators. II: Spectral theory. Selfadjoint operators in Hilbert space, Interscience, New York, 1963. MR 32 \#6181.

4. P. R. Halmos, A Hilbert space problem book, Van Nostrand, Princeton, N. J., 1967. MR 34 \#8178.

5. D. L. Skoug, Generalized Ilstow and Feynman integrals, Pacific J. Math. 26 (1968), 171-192. MR 37 \#6428.

UNIVERSITY OF NEBRASKa 